Claremont Colleges

Scholarship@ Claremont

All HMC Faculty Publications and Research

HMC Faculty Scholarship

4-1-2009

\title{
Counting on Chebyshev Polynomials
}

Arthur T. Benjamin

Harvey Mudd College

Daniel Walton '07

Harvey Mudd College

\section{Recommended Citation}

Benjamin, A.T., \& Walton, D. (2009). Counting on Chebyshev Polynomials. Mathematics Magazine, 82(2): 117-126.

This Article is brought to you for free and open access by the HMC Faculty Scholarship at Scholarship @ Claremont. It has been accepted for inclusion in All HMC Faculty Publications and Research by an authorized administrator of Scholarship @ Claremont. For more information, please contact scholarship@cuc.claremont.edu. 


\title{
Counting on Chebyshev Polynomials
}

\author{
Arthur T. Benjamin \\ Harvey Mudd College \\ Claremont, CA 91711 \\ benjamin@hmc.edu \\ Daniel Walton \\ UCLA \\ Los Angeles, CA 90095-1555 \\ waltond@ucla.edu
}

It's hard to avoid Chebyshev polynomials. They appear in just about every branch of mathematics, including geometry, combinatorics, number theory, differential equations, approximation theory, numerical analysis, and statistics. (Rivlin [6] gives numerous examples.) Their significance can be immediately appreciated by the fact that the function $\cos n \theta$ is a Chebyshev polynomial function of $\cos \theta$. Specifically, for $n \geq 0$,

$$
\cos (n \theta)=T_{n}(\cos (\theta)),
$$

where $T_{n}$ is the Chebyshev polynomial of the first kind, defined by $T_{0}(x)=1$, $T_{1}(x)=x$, and for $n \geq 2$,

$$
T_{n}(x)=2 x T_{n-1}(x)-T_{n-2}(x) .
$$

For example, $T_{2}(x)=2 x^{2}-1, T_{3}(x)=4 x^{3}-3 x, T_{4}(x)=8 x^{4}-8 x^{2}+1$. This generates the familiar trigonometric identity $\cos (2 \theta)=2 \cos ^{2} \theta-1$, and the less familiar $\cos (3 \theta)=4 \cos ^{3} \theta-3 \cos \theta$ and $\cos (4 \theta)=8 \cos ^{4} \theta-8 \cos ^{2} \theta+1$.

If we change the initial conditions to be $U_{0}(x)=1$ and $U_{1}(x)=2 x$, but keep the same recurrence

$$
U_{n}(x)=2 x U_{n-1}(x)-U_{n-2}(x),
$$

we get the Chebyshev polynomials of the second kind. For instance, $U_{2}(x)=4 x^{2}-1$, $U_{3}(x)=8 x^{3}-4 x, U_{4}(x)=16 x^{4}-12 x^{2}+1$.

The Chebyshev polynomials generate many fundamental sequences, including the constant sequence, the sequence of integers, and the Fibonacci numbers. It's easy to show that for all $n \geq 0, T_{n}(1)=1$ and $U_{n}(1)=n+1, T_{n}(-1)=(-1)^{n}$, $U_{n}(-1)=(-1)^{n}(n+1)$. When we substitute complex numbers, such as $x=i / 2$, the Fibonacci and Lucas numbers appear. Specifically,

$$
i^{-n} U_{n}(i / 2)=f_{n}
$$

and

$$
2 i^{-n} T_{n}(i / 2)=L_{n}
$$

where $f_{n}=f_{n-1}+f_{n-2}$, and $L_{n}=L_{n-1}+L_{n-2}$, with initial conditions $f_{0}=f_{1}=$ 1 , and $L_{0}=2, L_{1}=1$. (We note that the "classical" Fibonacci numbers are defined by $F_{0}=0$ and $F_{1}=1$, but $f_{n}=F_{n+1}$ is more natural for combinatorial purposes.) In fact, any sequence of numbers that satisfies a second order recurrence with constant coefficients can be expressed in terms of Chebyshev polynomials [1]. 
Here we list a few more intriguing identities satisfied by the Chebyshev polynomials. For $m, n \geq 0$,

$$
\begin{aligned}
U_{n}(x) & =\sum_{j=0}^{n} x^{j} T_{n-j}(x) \\
T_{m}\left(T_{n}(x)\right) & =T_{m n}(x)
\end{aligned}
$$

Finally, if we define $\hat{U}_{n}(x)=U_{n-1}(x)$, then

$$
\operatorname{gcd}\left(\hat{U}_{m}(x), \hat{U}_{n}(x)\right)=\hat{U}_{\operatorname{gcd}(m, n)}(x)
$$

All of the identities above can be proved by induction and various algebraic methods. The point of this article is to show that these identities, and many others, can also be given elegant combinatorial proofs, once we understand what the Chebyshev polynomials are counting.

\section{Combinatorial models for $U_{n}(x)$}

So what do Chebyshev polynomials count? As motivation, consider the combinatorial model for the Fibonacci numbers. It's easy to show $[3, \mathbf{4}]$, that the Fibonacci number $f_{n}$ counts the ways to tile a $1 \times n$ strip using $1 \times 1$ squares and $1 \times 2$ dominoes of length two. For example, $f_{4}=5$ counts the five tilings of length four below.

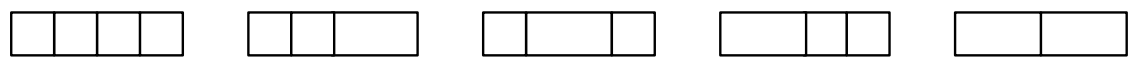

FIGURE 1: The fourth Fibonacci number $f_{4}=5$ is the number of square-domino tilings of length four

As it turns out, Chebyshev polynomials count the same objects as Fibonacci numbers, but we assign a weight to each tile. Specifically, we assign each square a weight of $2 x$ and each domino a weight of -1 , and define the weight of a tiling to be the product of the weights of its tiles. We provide the tilings of lengths two, three, and four, along with their respective weights, in FIGURE 2, and we see that their weights sum to Chebyshev polynomials, $U_{2}(x), U_{3}(x)$, and $U_{4}(x)$.

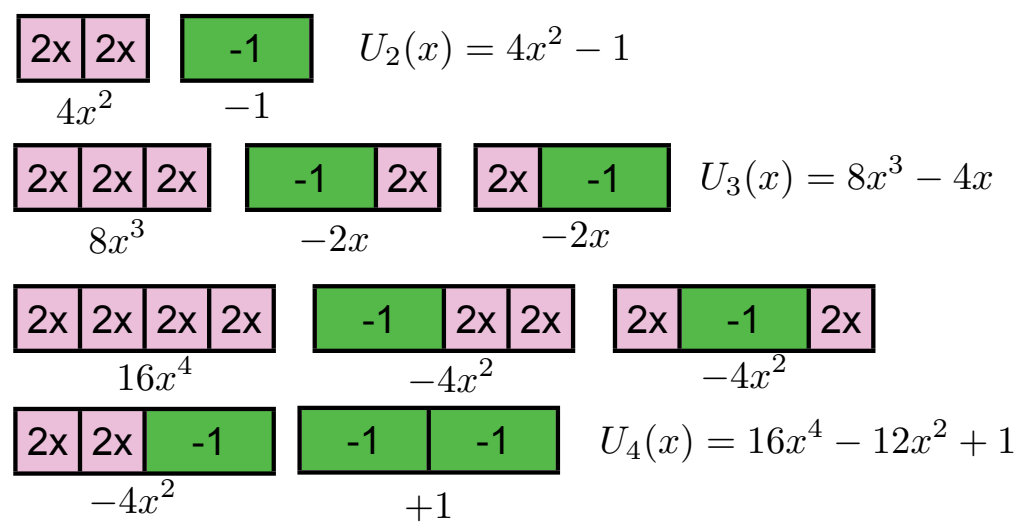

FIGURE 2: $U_{2}(x)=4 x^{2}-1, U_{3}(x)=8 x^{3}-4 x, U_{4}(x)=16 x^{4}-12 x^{2}+1$ is the total weight of all tilings of length two, three, and four, respectively

This suggests the following theorem, originally due to Louis Shapiro [7]. 
THEOREM 1. For $n \geq 0, U_{n}(x)$ is the sum of the weights of all square-domino tilings of length $n$.

Proof. Let $w_{n}$ denote the total weight of all tilings of length $n$. It's easy to verify that $w_{0}=1=U_{0}(x), w_{1}=2 x=U_{1}(x)$, and $w_{2}=4 x^{2}-1=U_{2}(x)$. Every tiling of length $n \geq 2$ comes from a tiling of length $n-1$ followed by a square (of weight $2 x$ ) or comes from a tiling of length $n-2$ followed by a domino (of weight -1 ). Hence, $w_{n}=2 x w_{n-1}-1 w_{n-2}$. Then, by induction and the recurrence for $U_{n}, w_{n}=$ $2 x U_{n-1}(x)-U_{n-2}(x)=U_{n}(x)$, as desired.

Notice that a tiling of length $n$ with $k$ dominoes has exactly $n-2 k$ squares and therefore has weight $(-1)^{k}(2 x)^{n-2 k}$. We leave it to the reader to show that the number of such tilings is $\left(\begin{array}{c}n-k \\ k\end{array}\right)$, which gives us the following closed form for $U_{n}(x)$.

IDENTITY 1. For $n \geq 0$,

$$
U_{n}(x)=\sum_{k=0}^{\lfloor n / 2\rfloor}\left(\begin{array}{c}
n-k \\
k
\end{array}\right)(-1)^{k}(2 x)^{n-2 k} .
$$

Moreover, if we let $x$ take on the imaginary value $x=i / 2$, we see that for $0 \leq k \leq$ $n / 2$, every length $n$ tiling with $k$ dominoes has weight $(-1)^{k} i^{n-2 k}=i^{n}$, independent of $k$. Since there are $f_{n}$ tilings of length $n$, we have $U_{n}(i / 2)=i^{n} f_{n}$, and therefore we have the following Fibonacci identity

IDENTITY 2. For $n \geq 0, i^{-n} U_{n}(i / 2)=f_{n}$.

The following "addition formula" is also easy to see, once we define the concept of breakability. We say that a tiling is unbreakable at cell $m$ if a domino covers cells $m$ and $m+1$; otherwise we say the tiling is breakable at cell $m$.

IDENTITY 3. For all $m, n \geq 1$,

$$
U_{m+n}(x)=U_{m}(x) U_{n}(x)-U_{m-1}(x) U_{n-1}(x) .
$$

Proof. The total weight of length $m+n$ tilings that are breakable at cell $m$ is $U_{m}(x) U_{n}(x)$ (by the distributive law). All tilings that are unbreakable at cell $m$ consist of a tiling of length $m-1$ followed by a domino (with weight -1 ) followed by a tiling of weight $n-1$, and thus have total weight $-U_{m-1}(x) U_{n-1}(x)$.

There is another way to interpret $U_{n}(x)$ combinatorially, which is a little more "colorful." Consider the set of colored tilings, where dominoes have just one color (light gray), but squares come in two colors (white or black). (Incidentally, the number of such tilings is the $n$th Pell number $p_{n}$, defined recursively by $p_{0}=1, p_{1}=2$ and for $n \geq 2, p_{n}=2 p_{n-1}+p_{n-2}$.) As in the previous model, we assign all dominoes a weight of -1 , but since $2 x=x+x$, we can assign each white square a weight of $x$ and each black square a weight of $x$. As before, the weight of a tiling is the product of the weights of its tiles. In FIGURE 3, we list the five colored tilings of length 2 and the twelve colored tilings of length 3 , along with their total weights.

Reasoning as before, we have the following theorem.

THEOREM 2. For $n \geq 0, U_{n}(x)$ is the sum of the weights of all colored squaredomino tilings of length $n$. 


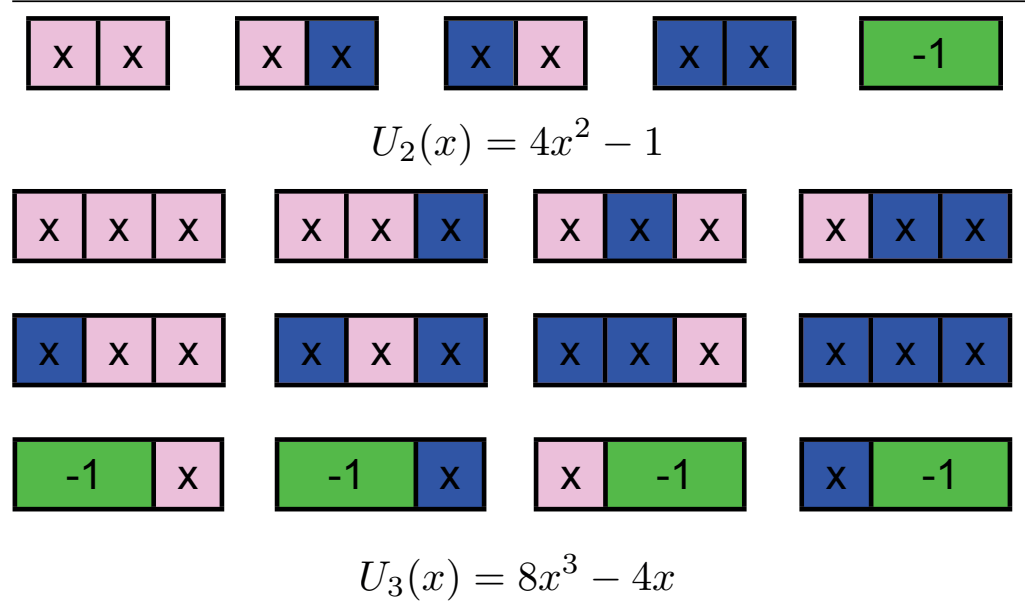

FIGURE 3: $U_{n}(x)$ using colored tilings, for $n=2$ and $n=3$

Having two colors of squares to play with will allow us to prove many interesting facts about Chebyshev polynomials (especially of the first kind). Here is a simple identity that is easy to prove by induction, but the combinatorial technique we introduce will be useful to us later on.

IDENTITY 4. For $n \geq 0, U_{n}(1)=n+1$.

Proof. If we assign all squares (black or white) a weight of $x=1$ and each domino, as usual, a weight of -1 , then the weight of a colored tiling will be $(-1)^{k}$, where $k$ is the number of dominoes. Consequently $U_{n}(1)$ counts the number of length $n$ tilings with an even number of dominoes minus the number of length $n$ tilings with an odd number of dominoes. Given any colored tiling $X$, we will try to pair it up with another tiling $X^{\prime}$ where the number of dominoes in $X$ and $X^{\prime}$ have opposite parity. (Or put more poetically, we try to find a mate of opposite weight.)

Given a tiling $X$ we look for the smallest number $k$ where either (i) cells $k$ and $k+1$ are covered by a domino or (ii) cell $k$ is covered by a white square and cell $k+1$ is covered by a black square. If case (i) occurs, then we define $X^{\prime}$ to be the same tiling as $X$ but with the first domino replaced by $w b$, where $w$ denotes a white square and $b$ denotes a black square. If case (ii) occurs, we replace the first $w b$ with a domino. Thus $X$ and $X^{\prime}$ have opposite weight. Notice that $\left(X^{\prime}\right)^{\prime}=X$, whenever $X^{\prime}$ is defined.

When is $X^{\prime}$ undefined? Whenever $X$ has no dominoes and no occurrence of $w b$, that is, whenever $X=b^{j} w^{n-j}$ ( $j$ black squares followed by $n-j$ white squares) for some $0 \leq j \leq n$. Thus, there are $n+1$ exceptional tilings, all of which have positive weight (since they have no dominoes), and therefore $U_{n}(1)=n+1$.

\section{Combinatorial models for $T_{n}(x)$}

Chebyshev polynomials of the first kind have at least four useful combinatorial interpretations using tilings. Since they satisfy the same recurrence as Chebyshev polynomials of the second kind, but with different initial conditions, then they only differ in how they weight the initial tile [4, Chapter 3]. As before, we define the weight of a length $n$ tiling of squares and dominoes to be the product of the weights of its tiles, where each domino has weight -1 and each square has weight $2 x$, but if the tiling 
begins with a square then that initial square has weight $x$. For example, the tilings for $T_{n}(x)$, with $n=2,3,4$ are given in FIGURE 4.

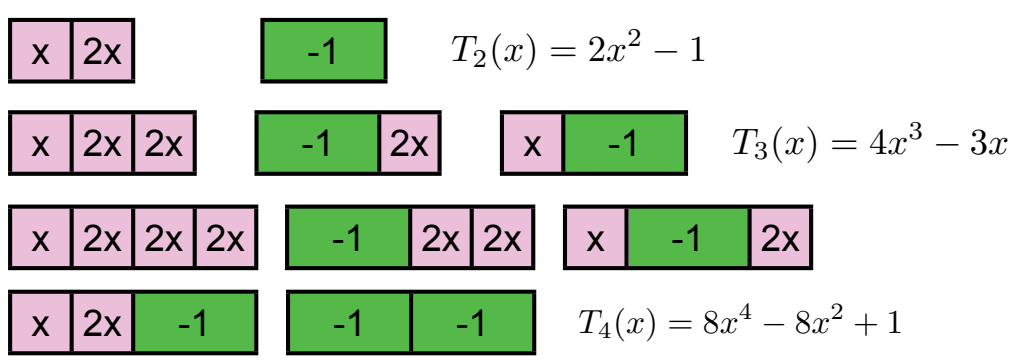

FIGURE 4: The weight of uncolored tilings as counted by $T_{n}(x)$ 1.

The following "uncolored" interpretation has essentially the same proof as Theorem

THEOREM 3. $T_{n}(x)$ is the total weight of all uncolored tilings of length $n$, where an initial square has weight $x$, all other squares have weight $2 x$, and all dominoes have weight -1 .

Reasoning as in Identity 3 , we get an addition formula for $T_{n}(x)$.

IDENTITy 5. For $m, n \geq 1, T_{m+n}(x)=T_{m}(x) U_{n}(x)-T_{m-1}(x) U_{n-1}(x)$.

We note that Identities 3 and 5 remain true when $m=0$ or $n=0$ provided we extend the recurrence so that $T_{-1}(x)=x$ and $U_{-1}(x)=0$.

Chebyshev polynomials of the first kind can also be thought of as counting circular tilings of bracelets. Specifically, if we take the previous model and multiply the weight of the initial tile by two, then all squares would receive a weight of $2 x$, but now an initial domino has weight -2 . We can think of this as counting two types of initial dominoes (each with weight -1 ). A domino of the first type will cover cells 1 and 2, as usual, but a domino of the second type will cover cells $n$ and 1, as in FIGURE 5, giving us the following theorem.

THEOREM 4. $2 T_{n}(x)$ is the total weight of all uncolored circular tilings of length $n$, where each square has weight $2 x$ and each domino has weight -1 .

By considering whether or not a bracelet has a domino covering cells $n$ and 1 , we have

IDENTITY 6. For $n \geq 1,2 T_{n}(x)=U_{n}(x)-U_{n-2}(x)$.

How many bracelets of length $n$ have exactly $k$ dominoes? By considering whether or not it has a domino covering cells $n$ and 1 , there are

$$
\left(\begin{array}{c}
n-k-1 \\
k-1
\end{array}\right)+\left(\begin{array}{c}
n-k \\
k
\end{array}\right)=\frac{n}{n-k}\left(\begin{array}{c}
n-k \\
k
\end{array}\right)
$$

such bracelets. Thus by the reasoning that precedes Identity 1 , we have a similar closed form for $T_{n}(x)$.

IDENTITY 7. For $n>0$,

$$
T_{n}(x)=\frac{1}{2} \sum_{k=0}^{\lfloor n / 2\rfloor} \frac{n}{n-k}\left(\begin{array}{c}
n-k \\
k
\end{array}\right)(-1)^{k}(2 x)^{n-2 k} .
$$



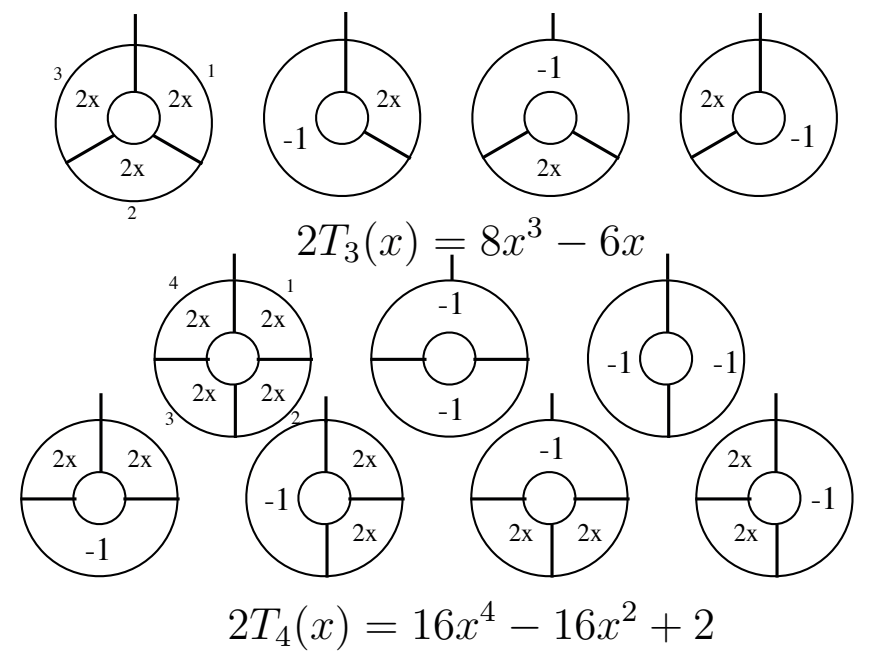

FIGURE 5: $2 T_{n}(x)$ counts weighted bracelets

Since the number of length $n$ bracelets is well known to be the Lucas number $L_{n}$ [4, Chapter 2]. So just like with Identity 2, we have

IDENTITy 8. For $n \geq 0,2 i^{-n} T_{n}(i / 2)=L_{n}$.

Other useful identities are obtained by allowing our squares to come in two colors. As an immediate consequence of Theorem 3, we have

THEOREM 5. $T_{n}(x)$ is the total weight of all colored tilings of length $n$, where an initial square has weight $x / 2$, all other squares have weight $x$, and all dominoes have weight -1 .

Alternatively, we can allow all squares, to have weight $x$, but now we restrict the color of an initial square.

THEOREM 6. $T_{n}(x)$ is the total weight of all colored tilings of length $n$, where all squares have weight $x$, all dominoes have weight -1 , but the tiling may not begin with a black square. (Alternatively, $T_{n}(x)$ counts tilings that do not begin with a white square.)

In Figure 6, we list the restricted colored tilings counted by $T_{n}(x)$, for $n=2$ and $n=3$.
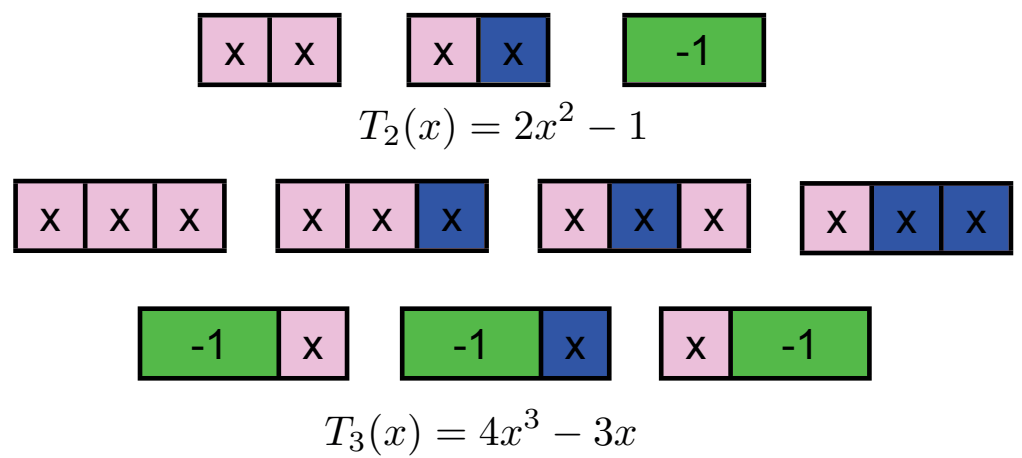

FIGURE 6: Examples of $T_{n}(x)$ using restricted colored tilings 
This tiling interpretation of $T_{n}(x)$ was exploited combinatorially by Walton [8] to prove many Chebyshev polynomial identities. For example, by applying the same argument as done in Identity 4, we invite the reader to combinatorially prove

IDENTITY 9. For all $n \geq 0, T_{n}(1)=1$.

Here is another quick proof of something we call the "string of lights" identity.

IDENTITY 10. For $n \geq 1, T_{n}(x)=\sum_{j=1}^{n}\left(x^{j} T_{n-j}(x)\right)-U_{n-2}(x)$

Proof. A restricted tiling must either begin with a domino or a string of white squares. The total weight of tilings of length $n$ that begin with exactly $j \geq 1$ white squares is $x^{j} T_{n-j}(x)$ since the tile that follows the first $j$ white squares is restricted to be a domino or a dark square. The total weight of those tilings that begin with a domino is $-U_{n-2}(x)$ since the initial domino has weight -1 and the remaining colored tiling is unrestricted.

We invite the reader to combinatorially prove

IDENTITY 11. For $n \geq 1, U_{n}(x)=T_{n}(x)+x U_{n-1}(x)$.

With more ingenuity, Walton $[8]$ presents combinatorial proofs of trickier identities. For example, for $n \geq m \geq 0$,

$$
T_{n}^{2}(x)+T_{m}^{2}(x)=1+T_{n+m}(x) T_{n-m}(x),
$$

and for $m, n \geq 0$,

$$
T_{m}\left(T_{n}(x)\right)=T_{m n}(x) .
$$

We will come back to this last identity in the next section.

At this point, we should expose the fact that some of the identities presented here are true for any sequence satisfying a second order recurrence with constant coefficients. If $u_{-1}=0, u_{0}=1$, and $u_{n}$ satisfies the recurrence $u_{n}=a u_{n-1}+b u_{n-2}$, then $u_{n}$ is the total weight of all tilings of length $n$ where squares have weight $a$, dominoes have weight $b$, and the weight of a tiling is the product of its weights [3]. (Ironically, these are called Lucas sequences of the first kind, but they correspond to Chebyshev polynomials of the second kind.) Thus we immediately obtain generalizations of some of our earlier identities like

$$
u_{n}=\sum_{k=0}^{\lfloor n / 2\rfloor}\left(\begin{array}{c}
n-k \\
k
\end{array}\right) b^{k} a^{n-2 k}
$$

and

$$
u_{m+n}=u_{m} u_{n}+b u_{m-1} u_{n-1} .
$$

The constants $a$ and $b$ can be real or complex numbers, but they could also be polynomials. Moreover if $a$ and $b$ are relatively prime integers, and if we define $U_{n}=u_{n-1}$, then it can be shown by combinatorial argument [3] that

$$
\operatorname{gcd}\left(U_{m}, U_{n}\right)=U_{\operatorname{gcd}(m, n)} .
$$

The same line of reasoning will work when $a$ and $b$ are relatively prime polynomials like $2 x$ and -1 , which explains equation (7) in the introduction. 


\section{Combinatorial trigonometry}

Finally, we come full circle and explain the trigonometric identity (1) at the beginning of the paper, namely

$$
\text { IDENTITY 12. For } n \geq 0, \cos (n \theta)=T_{n}(\cos \theta) \text {. }
$$

Readers may wish to prove this theorem by induction, using the definition of $T_{n+1}(x)$ and two applications of the angle addition formula for cosine. But the combinatorial proof, due to Benjamin, Ericksen, Jayawant, and Shattuck [2], is more fun and leads to other insights.

Proof. From Theorem 3, $T_{n}(\cos \theta)$ is the total weight of all tilings of length $n$ where each domino has weight -1 and each square has weight $2 \cos \theta$, except for an initial square, which has weight $\cos \theta$. But how do we $\operatorname{combinatorialize} \cos \theta$ ? First, we use a formula from Euler

$$
\cos \theta=\frac{e^{i \theta}+e^{-i \theta}}{2}
$$

Then we assign to each square, except for an initial one, the weight $e^{i \theta}+e^{-i \theta}$, and half that weight to an initial square. Next, we introduce colored squares, but this time the white and black squares are given different weights: the weight of a white square is $e^{i \theta}$ and the weight of a black square is $e^{-i \theta}$ (except for an initial colored square, whose weight will be $\frac{1}{2} e^{i \theta}$ or $\frac{1}{2} e^{-i \theta}$ ). Thus, for example, the colored tiling in FIGURE 7 has weight $\frac{1}{2} e^{3 i \theta}$.

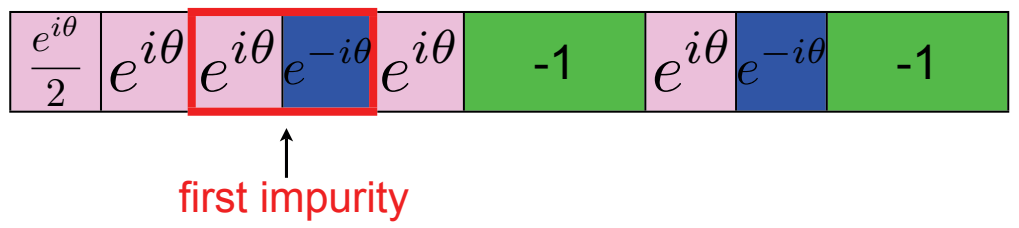

FIGURE 7: A colored tiling with weight $\frac{1}{2} e^{3 i \theta}$

Reasoning as in Theorem 5, $T_{n}(\cos \theta)$ is the sum of the weights of all of these colored tilings. Our goal is to show that this sum is nearly zero. We say that a colored tiling is impure if it contains two consecutive square tiles of opposite color or at least one domino. In a colored tiling, we will call a domino or two consecutive squares of opposite color an impurity. For example, the tiling in FIGURE 7 has its first impurity on cells 3 and 4 .

Next we claim that the sum of the weights of all impure tilings is zero. Let $X$ be an impure tiling with its first impurity on cells $k$ and $k+1$. We consider two cases.

First consider the case where $k \geq 2$. If cells $k$ and $k+1$ are squares of opposite color, then we "find a mate of opposite weight" $X^{\prime}$ by replacing those two squares with a domino and leave all other tiles the same. If cells $k$ and $k+1$ are covered by a domino, then we form $X^{\prime}$ by replacing the domino with two squares of opposite color where the color of the square on cell $k$ is the same as the color of the square on cell $k-1$. Thus $\left(X^{\prime}\right)^{\prime}=X$. Moreover, since two squares of opposite color have a weight $e^{i \theta} e^{-i \theta}=1$ and a domino has weight -1 , then it is clear that $X$ and $X^{\prime}$ have opposite weight, so their weights sum to zero, as in FIGURE 8.

On the other hand, if $k=1$, then we "find a trio that sums to zero" by creating tilings $X_{1}$ and $X_{2}$, which are identical to $X$ except for the first two cells. Among $X$, 


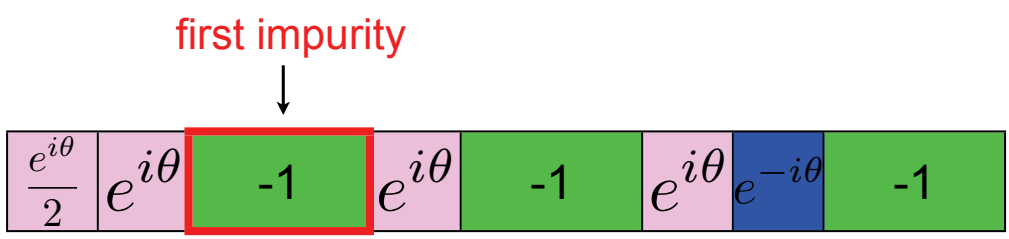

FIGURE 8: This tiling $X^{\prime}$ is the mate of the previous one and has weight $-\frac{1}{2} e^{3 i \theta}$

$X_{1}$ and $X_{2}$, one of them begins with $w b$ (with weights that multiply to $1 / 2$ ) one of them begins with $b w$ (with weights that multiply to $1 / 2$ ) and the other begins with a domino (with weight -1 ). Thus the weights of $X, X_{1}$ and $X_{2}$ sum to zero.

Consequently, every impure tiling belongs to a pair or trio with weights that sum to zero. Thus $T_{n}(\cos \theta)$ is just the sum of the weights of the pure tilings. But there are only two pure tilings, namely the tiling consisting of $n$ white squares, with weight $e^{i n \theta} / 2$, and the tiling consisting of all black squares, with weight $e^{-i n \theta} / 2$. Thus

$$
T_{n}(\cos \theta)=\frac{e^{i n \theta}+e^{-i n \theta}}{2}=\cos (n \theta),
$$

as desired.

By the same logic, we can generalize the last identity as follows.

IDENTITY 13. For $n \geq 0$ and any real or complex number $z \neq 0$,

$$
T_{n}\left(\frac{z+1 / z}{2}\right)=\frac{z^{n}+1 / z^{n}}{2} .
$$

We note that once the theorem is expressed in this form, it can then be proved easily by induction, but the combinatorial proof allows us to anticipate and appreciate this generalization.

By a slightly different argument and using $\sin \theta=\frac{e^{i \theta}-e^{-i \theta}}{2 i}$, we can prove

$$
\sin ((n+1) \theta)=U_{n}(\cos \theta) \sin \theta
$$

and its generalization

$$
\left(\frac{z-1 / z}{2}\right) U_{n}\left(\frac{z-1 / z}{2}\right)=\frac{z^{n}-1 / z^{n}}{2} .
$$

These and other trigonometric identities can also be given combinatorial proofs [2].

Identity 12 also leads to a quick proof of the composition theorem mentioned in the introduction.

IDENTITY 14. For $m, n \geq 0, T_{m}\left(T_{n}(x)\right)=T_{m n}(x)$.

Proof. When $x=\cos \theta$, we have

$$
T_{m}\left(T_{n}(\cos \theta)\right)=T_{m}(\cos (n \theta))=\cos (m n \theta)=T_{m n}(\cos \theta) .
$$

Since these polynomials agree at an infinite number of points (namely for all points $\cos \theta$ ), then they must be the same polynomial.

Using a similar argument, there is a composition theorem for the Chebyshev polynomials of the second kind, namely

$$
U_{m-1}\left(T_{n}(x)\right) U_{n-1}(x)=U_{m n-1}(x) .
$$


For the combinatorial proof enthusiast, both of these composition theorems can also be proved using "tilings of tilings" $[\mathbf{5 , 8}]$, but some might say that this is going a little "overboard."

Acknowledgment. The authors are grateful to the referees for their many helpful suggestions.

\section{REFERENCES}

1. D. Aharonov, A. Beardon, and K. Driver, Fibonacci, Chebyshev, and Orthogonal Polynomials, Amer. Math. Monthly, 112:7 (August-September 2005) 612-630.

2. A. T. Benjamin, L. Ericksen, P. Jayawant, and M. Shattuck, Combinatorially Composing Chebyshev Polynomials, to appear in Journal of Statistical Planning and Inference, Proceedings of the 6th International Conference on Lattice Path Combinatorics, 2008.

3. A. T. Benjamin and J. J. Quinn, The Fibonacci Numbers-Exposed More Discretely, this Magazine, 76:3 (June 2003) 182-192.

4. A. T. Benjamin and J. J. Quinn, Proofs That Really Count: The Art of Combinatorial Proof, Mathematical Association of America, Washington DC, 2003.

5. A. T. Benjamin and D. Walton, Combinatorially Composing Chebyshev Polynomials, to appear in Journal of Statistical Planning and Inference, Proceedings of the 6th International Conference on Lattice Path Combinatorics, 2008.

6. T. J. Rivlin, Chebyshev Polynomials: From Approximation Theory to Algebra and Number Theory, John Wiley and Sons, New York, 1990.

7. L. W. Shapiro, A Combinatorial Proof of a Chebyshev Polynomial Identity, Discrete Math. 34 (1981) 203-206.

8. D. Walton, A Tiling Approach to Chebyshev Polynomials, Senior Thesis, Harvey Mudd College, Claremont, CA, 2007. 